Article

\title{
Prospective Associations of Serum Adiponectin, Leptin, and Leptin-Adiponectin Ratio with Incidence of Metabolic Syndrome: The Korean Genome and Epidemiology Study
}

\author{
Kyung Won Lee ${ }^{1(1)}$ and Dayeon Shin ${ }^{2, *(1)}$ \\ 1 Department of Food Science and Nutrition, Gwangju University, Gwangju 61743, Korea; \\ kwlee@gwangju.ac.kr \\ 2 Department of Food and Nutrition, Inha University, Incheon 22212, Korea \\ * Correspondence: dyshin@inha.ac.kr; Tel.: +82-32-860-8123
}

Received: 31 March 2020; Accepted: 5 May 2020; Published: 8 May 2020

check for updates

\begin{abstract}
Although the role of adiponectin and leptin in the etiology of metabolic syndrome (MetS) has been explored in various populations, limited knowledge is available on the prospective association of adiponectin and leptin with the risk of MetS development. The present study aimed to evaluate the associations of adiponectin, leptin, and the leptin-adiponectin (LA) ratio with the future risk of MetS in middle-aged and older Korean adults. Using a prospective, population-based Ansan-Ansung cohort of the Korean Genome and Epidemiology Study (KoGES), 2691 Korean adults (1317 men and 1374 women) were included in the present study. Serum adiponectin and leptin concentrations were measured using commonly available enzyme-linked immunosorbent assay kits. Multivariable Cox proportional hazard models were used to investigate the relationships of the different adiponectin and leptin concentrations and LA ratio with the incident MetS. During a mean follow-up of 6.75 years, a total of $359(27.26 \%)$ men and $385(28.02 \%)$ women were identified as developing new-onset MetS. After controlling for covariates, higher adiponectin levels were associated with lower incidence of MetS (hazard ratio (HR) for third vs. first tertile: $0.53,95 \%$ confidence interval (CI): $0.40-0.70$ for men and HR: 0.54, 95\% CI: 0.42-0.71 for women), while higher leptin levels (HR for third vs. first tertile: $2.88,95 \%$ CI: $2.01-4.13$ for men and HR: $1.55,95 \%$ CI: $1.13-2.13$ for women) and LA ratio (HR for third vs. first tertile: 3.07, 95\% CI: 2.13-4.44 for men and HR: 1.94, 95\% CI: 1.41-2.66 for women) were associated with an increased incidence of MetS. Among men, in the fully adjusted models an increase by one standard deviation (SD) in adiponectin levels was associated with a $10 \%$ decrease in MetS risk (HR per SD: 0.90, 95\% CI: 0.85-0.95) while leptin and LA ratio was associated with a $5 \%$ (HR per SD: 1.05, 95\% CI: 1.01-1.08) and 40\% (HR per SD: 1.40, 95\% CI: 1.22-1.62) increase in MetS risk, respectively. Among women, a significant association with MetS risk was observed only in adiponectin levels (HR per SD: 0.91, 95\% CI: 0.88-0.95). We found that higher adiponectin level was associated with a lower risk of MetS, while higher leptin level and LA ratio were associated with elevated MetS incidence, irrespective of body mass index at baseline in both Korean men and women. Adiponectin and leptin levels and LA ratio could play a role as a useful biomarker in the prediction of future MetS development among middle-aged and older Koreans.
\end{abstract}

Keywords: adiponectin; leptin; metabolic syndrome; prospective study; Koreans

\section{Introduction}

In contrast to three or four decades ago when infectious diseases were the main cause of death, the healthcare burden from non-communicable diseases (NCDs) continues to rise worldwide [1]. Following 
this trend, Korea is also encountering a rising level of NCD cases. Deaths due to NCDs account for $80 \%$ of all deaths in Korea, accounting for seven of the top 10 causes of death in the country [2]. NCDs include a variety of diseases, but one disease in particular that poses many challenges is metabolic syndrome (MetS). MetS is characterized as a cluster of metabolic conditions, including abdominal obesity, dyslipidemia, elevated fasting blood glucose, and high blood pressure [3].

According to the Metabolic Syndrome Fact Sheet in Korea 2018, one out of five Korean adults has MetS. Despite various efforts to prevent MetS, the prevalence of the disease, rising from $21.1 \%$ in 2007 to $22.4 \%$ in 2015, has not exhibited any signs of a significant decrease [4]. In addition, MetS prevalence has drastically increased for both men and women in their 50s and older. Furthermore, as MetS is known as a key risk factor for cardiovascular disease and diabetes, great emphasis is being placed on the importance of preventing and treating MetS $[5,6]$.

As with other chronic diseases, it is necessary to identify the biomarkers associated with MetS occurrence in order to aid prevention efforts via screening of high-risk groups. Several clinically relevant biomarkers associated with MetS have been proposed, specifically adiponectin and leptin [7]. As members of the adipokine family of cytokines secreted from the adipose tissue, adiponectin and leptin carry out antagonistic functions in the body [8]. Adiponectin is involved in energy metabolism and exhibits anti-inflammatory effects, including its role as an insulin sensitizer [9]. Low adiponectin levels are found in obese individuals, and is associated with insulin resistance, diabetes, and cardiovascular disease $[10,11]$. Leptin, however, aids the regulation of appetite by producing a feeling of satiation when food is ingested, and maintains fat storage in the body at a consistent level [12]. Leptin is associated with pro-inflammatory effects, including regulation of the secretion of cytokines such as tumor necrosis factor- $\alpha$ and interleukin-6. Elevated leptin levels can also cause obesity, diabetes, and heart disease $[13,14]$. In addition, hyperleptinemia, rather than hyperinsulinemia or insulin resistance, has been reported as a critical risk factor for MetS [15].

Despite active research on the role of adiponectin and leptin with regards to MetS risk for diverse ethnic groups [16-18], investigations into the association of adiponectin and leptin for MetS risk in Korea have been limited to cross-sectional studies [19,20] or short-term follow-up studies [21,22]. Previous studies have mostly been conducted with people in the Western hemisphere, who have a different adiposity and body composition to Asian populations. Considering that the concentration of adiponectin and leptin in the blood is affected by the level of obesity [10,23], and the lower relative adiposity seen in Korean compared with Western populations, research revealing the prospective association of adiponectin and leptin to MetS risk in a Korean population, observed over an extended follow-up period, is needed with a relatively lower adiposity than Westerners, considering the effect of obesity. Therefore, the present study aimed to investigate the association of three markers-adiponectin, leptin, and the leptin-adiponectin (LA) ratio-with the risk of MetS development in middle-aged and older Korean adults, an age group experiencing a relatively drastic increase in the prevalence of MetS.

\section{Methods}

\subsection{Data Source and Study Population}

We used data from a prospective, population-based Ansan-Ansung cohort study, part of the Korean Genome and Epidemiology Study (KoGES) conducted by the Korea National Institute of Health (KNIH). In this study, 10,030 Korean adults aged 40-69 years were recruited from two Korean cities (Ansan and Ansung), and were followed up biennially over a 14-year period. Detailed study design and procedures of the Ansan-Ansung cohort study have been previously published [24]. The KNIH additionally identified 15 biomarkers, including adiponectin and leptin, using the stored blood samples obtained from individuals who participated in the third follow-up examination. Since the third follow-up examination provided information on serum adiponectin and leptin levels, we performed the analysis by considering this data as the baseline. In total, 6688 people participated in the third follow-up examination between 2007 and 2008, among whom 1100 were excluded due to an absence of 
stored blood samples. Of the remaining 5578 participants, those with existing metabolic syndrome $(n=2822)$, any type of cancer $(n=45)$, or missing information on covariates $(n=20)$ were also excluded (Figure 1). Final subjects included in the analysis comprised 2691 Korean adults (1317 men and 1374 women), followed up until 2016. The Institutional Review Board of the Korea Centers for Disease Control and Prevention reviewed and approved the KoGES study protocol. Each participant's written informed consent was obtained in accordance with the relevant institutional guidelines and regulations. The study was approved by the Institutional Review Board of Gwangju University (IRB number: 2-1041318-A-N-01-202001-HR-001-01). The dataset of Ansan-Ansung cohort used in this study are available through online sharing service after review of the research plan by the Korea Centers for Disease Control and Prevention.

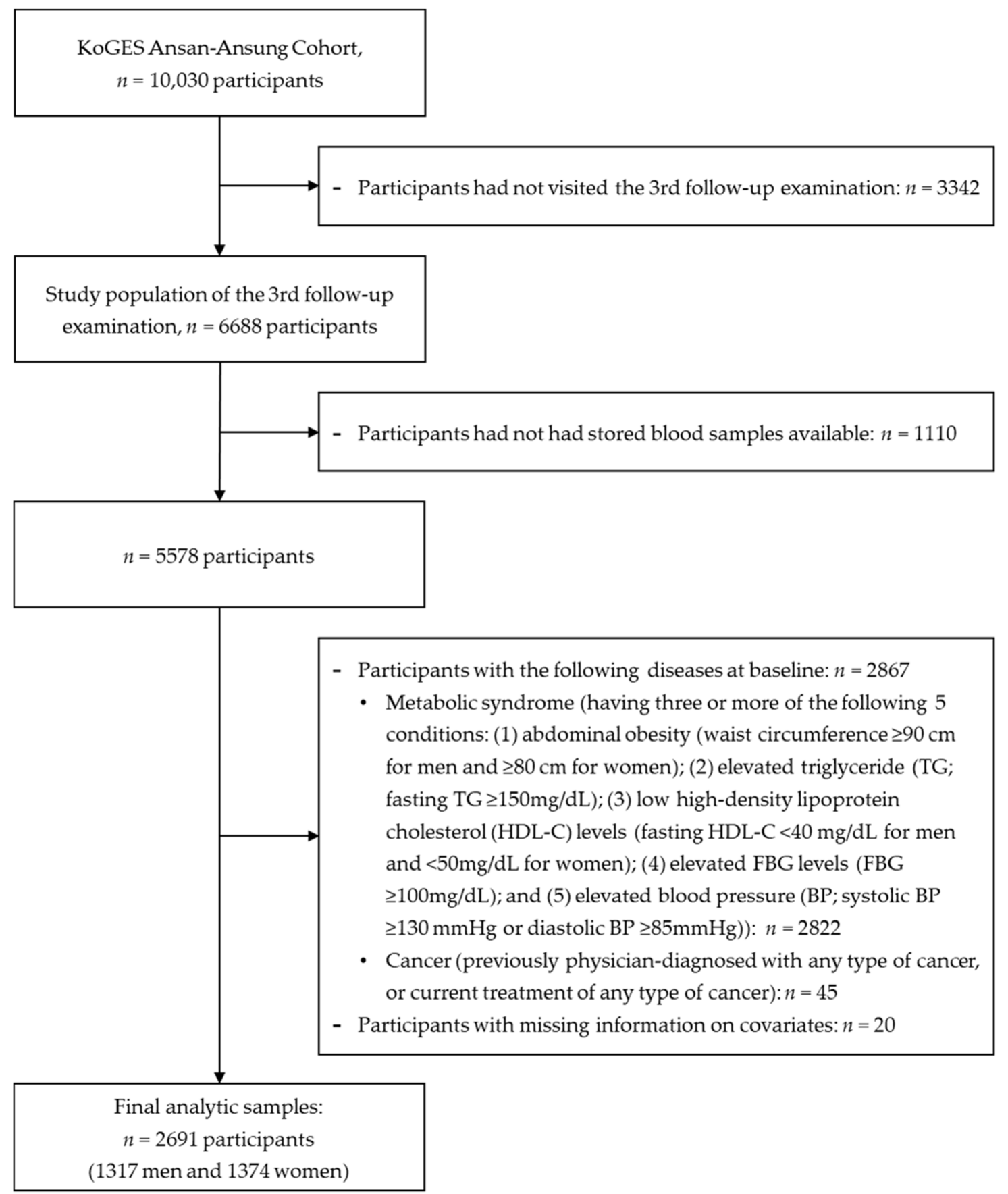

Figure 1. Flow diagram of the study population. 


\subsection{Measurements of Anthropometric and Biochemical Parameters}

All participants visited the survey site for an interview and clinical assessments at baseline and each follow-up. Information on sociodemographic characteristics, lifestyle, medical history, anthropometric measures, and biospecimens were collected by trained staff using standardized protocols during each examination, and participants of the cohort were followed-up biennially. Height, weight, and waist circumference were measured using standard methods in light clothing and barefoot. Body mass index (BMI) was calculated based on measured height and weight (body mass $(\mathrm{kg}) /$ height $\left(\mathrm{m}^{2}\right)$ ). Blood pressure (BP) was measured by trained technicians using a conventional mercury sphygmomanometer (Baumanometer; Baum, Copiague, NY, USA). Systolic BP and diastolic BP were measured on both arms in the sitting position after at least 5 min of rest. The average of measurements on the left and right arms were used to define values of systolic BP and diastolic BP in this study. For the measurement of biochemical components, all blood samples were taken after fasting for $12 \mathrm{~h}$. Serum adiponectin and serum leptin concentrations were measured using commonly available enzyme-linked immunosorbent assay (ELISA) kits (Mesdia, Seoul, Korea). Levels of serum adiponectin, serum leptin, and the corresponding LA ratio were analyzed as tertiles (tertile cut-offs were based on the sex-specific distributions of the main exposure variables) and continuous measures. Serum blood glucose, triglyceride (TG), high-density lipoprotein cholesterol (HDL-C) were assessed enzymatically using a Hitachi 747 chemistry analyzer (Hitachi, Tokyo, Japan). Serum high-sensitivity C-reactive protein (hs-CRP) was measured by enzymatic colorimetric methods using an automatic analyzer (ADVIA 1650 and 1800, Siemens, Tarrytown, NY, USA).

\subsection{Ascertainment of Metabolic Syndrome}

Diagnosis of MetS was carried out according to the National Cholesterol Education Program Adult Treatment Panel III (NCEP ATP III) [25] and International Diabetes Federation [26]. Based on these parameters, new-onset MetS is identified in this study by the presence of three or more of the following conditions: (1) abdominal obesity (waist circumference $\geq 90 \mathrm{~cm}$ for men and $\geq 80 \mathrm{~cm}$ for women); (2) elevated BP (systolic BP $\geq 130 \mathrm{mmHg}$ or diastolic $\mathrm{BP} \geq 85 \mathrm{mmHg}$ ); (3) elevated fasting blood glucose ((FBG) $\geq 100 \mathrm{mg} / \mathrm{dL}$ ); (4) elevated TG (fasting TG $\geq 150 \mathrm{mg} / \mathrm{dL}$ ); and (5) HDL-C levels (fasting HDL-C $<40 \mathrm{mg} / \mathrm{dL}$ for men and $<50 \mathrm{mg} / \mathrm{dL}$ for women). In the survival analyses, survival time for individuals who reported any diagnosis of MetS during the follow-ups was defined as the time between the baseline and the date of the new onset of MetS. Participants who were not diagnosed with MetS until the end of the study were censored on the date of the last follow-up examination, and survival time was defined as the time between the baseline and the last follow-up.

\subsection{Statistical Analyses}

All analyses were performed for men and women separately because women had higher adiponectin and leptin levels than men. According to the statistical distribution of three indicators, we split our study population into sex-specific tertiles of serum adiponectin (cut-offs for 4.35, $5.94 \mu \mathrm{g} / \mathrm{mL}$ for men and 5.88, $8.09 \mu \mathrm{g} / \mathrm{mL}$ for women), leptin (cut-offs for 1.38, $2.91 \mathrm{ng} / \mathrm{mL}$ for men and 7.36, $12.63 \mathrm{ng} / \mathrm{mL}$ for women), and LA ratio (cut-offs for 0.263, $0.643 \mathrm{ng} / \mu \mathrm{g}$ for men and 0.990, $2.016 \mathrm{ng} / \mathrm{\mu g}$ for women). Descriptive analysis of the baseline characteristics of the study population according to tertiles of adiponectin, leptin, and LA ratio were presented as frequencies (percentages) for categorical variables and means \pm standard deviations (SDs) or medians (interquartile ranges) for continuous variables. Baseline characteristics according to tertiles of three biomarkers were compared using chi-square tests, generalized linear regressions, and Kruskal-Wallis tests. We investigated the relationships between the different adiponectin and leptin concentrations and the LA ratio with incident MetS and its components to calculate hazard ratios (HRs) and 95\% confidence intervals (95\% CIs) using multivariable Cox proportional hazard models. Two models were applied: model 1 was adjusted for age (years); and model 2 was further adjusted for area of residence (Ansan or Ansung), education level (elementary 
school or lower, middle/high school, or college or higher), smoking status (never, past, or current), alcohol consumption (g/d), regular physical activity (yes or no), BMI $\left(\mathrm{kg} / \mathrm{m}^{2}\right)$, family history of diabetes (yes or no), and serum hs-CRP levels (mg/L). To test the linear trend, median values of each tertile group of main exposure variables were assigned to the regression models as a continuous variable. To investigate the joint effect of adiponectin and leptin concentrations on the development of MetS, individuals were cross-classified by sex-specific tertiles of serum adiponectin and leptin levels into 9 groups, and the combination of high adiponectin and low leptin levels was considered as the referent. Data management and statistical analyses were performed with SAS software version 9.4 (SAS Institute, Inc., Cary, NC, USA). A two-sided $P$-value of $<0.05$ was considered to be statistically significant.

\section{Results}

During a mean follow-up of $6.75 \pm 2.11$ years (18,162 person-years), a total of $359(27.26 \%)$ men and $385(28.02 \%)$ women were identified with new-onset MetS. Table 1 describes the characteristics of the study population according to LA ratio at baseline. In both men and women, individuals in the highest adiponectin tertile were more likely to be young, highly educated, and reside in rural areas (all, $P<0.05$ ). Compared to those in the lowest tertile of adiponectin, those in the highest tertile had a higher average BMI, higher average leptin level and LA ratio. The prevalence of individual components of MetS differed significantly by adiponectin levels. Participants in the highest adiponectin tertile showed a higher proportion of abdominal obesity, elevated TG, and low HDL-C at baseline examination $(P<0.001)$. In addition, most of participants in the highest adiponectin tertile had at least one risk factor for MetS. Baseline characteristics of study participants according to tertiles of adiponectin and leptin are shown in Supplementary Tables S1 and S2, respectively.

Tables 2 and 3 summarize significant associations of adiponectin, leptin, and LA ratio with 10-year MetS incidence in men and women. When models were controlled for age, area of residence, education level, smoking status, alcohol consumption, regular physical activity, BMI, family history of diabetes, and serum hs-CRP levels (model 2), higher adiponectin levels were associated with a lower incidence of MetS (HR for third vs. first tertile of adiponectin: 0.53, 95\% CI: 0.40-0.70, $P_{\text {trend }}<0.0001$ for men; 0.54, 95\% CI: $0.42-0.71, P_{\text {trend }}<0.0001$ for women), while higher leptin levels and LA ratio were associated with increased incidence of MetS (HR for third vs. first tertile of leptin: 2.88, 95\% CI: 2.01-4.13, $P_{\text {trend }}<0.0001$ for men; $1.55,95 \%$ CI: 1.13-2.13, $P_{\text {trend }}<0.0001$ for women; HR for third vs. first tertile of LA ratio: $3.07,95 \%$ CI: $2.13-4.44, P_{\text {trend }}<0.0001$ for men and $1.94,95 \%$ CI: $1.41-2.66, P_{\text {trend }}<0.0001$ for women). Among men, in the fully adjusted models, an increase by one SD in adiponectin levels was associated with a 10\% decrease in risk of MetS (HR per SD: 0.90, 95\% CI: 0.85-0.95), while leptin and LA ratio was associated with a 5\% (HR per SD: $1.05,95 \%$ CI: $1.01-1.08$ ) and $40 \%$ (HR per SD: $1.40,95 \%$ CI: 1.22-1.62) increase in MetS risk, respectively. Among women, a significant and inverse association with MetS risk was observed only for adiponectin levels (HR per SD: 0.91, 95\% CI: 0.88-0.95). 
Table 1. Characteristics of the study participants according to leptin-adiponectin ratios in Korean men and women aged 45-76 years.

\begin{tabular}{|c|c|c|c|c|c|c|c|c|}
\hline & \multicolumn{4}{|c|}{ Men } & \multicolumn{4}{|c|}{ Women } \\
\hline & \multicolumn{3}{|c|}{ Tertile of LA Ratio $^{1}$} & \multicolumn{5}{|c|}{ Tertile of LA Ratio } \\
\hline & $\begin{array}{l}\text { T1 (Lowest) } \\
(\mathrm{n}=439)\end{array}$ & $\begin{array}{c}\text { T2 } \\
(n=439)\end{array}$ & $\begin{array}{l}\text { T3 (Highest) } \\
(\mathrm{n}=439)\end{array}$ & $P^{3}$ & $\begin{array}{l}\text { T1 (Lowest) } \\
(\mathrm{n}=458)\end{array}$ & $\begin{array}{c}\text { T2 } \\
(n=458)\end{array}$ & $\begin{array}{l}\text { T3 (Highest) } \\
(\mathrm{n}=458)\end{array}$ & $P$ \\
\hline Age (years) & $59.07 \pm 8.53^{2}$ & $56.63 \pm 8.51$ & $54.53 \pm 7.68$ & $<0.0001$ & $56.84 \pm 8.62$ & $53.86 \pm 6.95$ & $53.06 \pm 6.70$ & $<0.0001$ \\
\hline Area of residence & & & & $<0.0001$ & & & & $<0.0001$ \\
\hline Ansan & $245(55.81)$ & $180(41.00)$ & $121(27.56)$ & & $230(50.22)$ & $162(35.37)$ & $124(27.07)$ & \\
\hline Ansung & $194(44.19)$ & $259(59.00)$ & $318(72.44)$ & & $228(49.78)$ & $296(64.63)$ & $334(72.93)$ & \\
\hline Education level & & & & $<0.0001$ & & & & $<0.0001$ \\
\hline selementary school & $102(23.23)$ & $82(18.68)$ & $38(8.66)$ & & $157(34.28)$ & $122(26.64)$ & $98(21.40)$ & \\
\hline middle/high school & $260(59.23)$ & $254(57.86)$ & $265(60.36)$ & & $266(58.08)$ & $292(63.76)$ & $311(67.90)$ & \\
\hline$\geq$ college & $77(17.54)$ & $103(23.46)$ & $136(30.98)$ & & $35(7.64)$ & $44(9.61)$ & $49(10.70)$ & \\
\hline Smoking status & & & & 0.0688 & & & & 0.4408 \\
\hline Never & $114(25.97)$ & $134(30.52)$ & $114(25.97)$ & & $452(98.69)$ & $451(98.47)$ & $449(98.03)$ & \\
\hline Past & $151(34.40)$ & $170(38.72)$ & $193(43.96)$ & & $2(0.44)$ & $3(0.66)$ & $3(0.66)$ & \\
\hline Current & $174(39.64)$ & $135(30.75)$ & $132(30.07)$ & & $4(0.87)$ & $4(0.87)$ & $6(1.31)$ & \\
\hline $\begin{array}{l}\text { Alcohol consumption } \\
\text { (grams/day) }^{4}\end{array}$ & $4.70(0.00-18.81)$ & $4.70(0.00-20.26)$ & $10.13(0.60-25.25)$ & 0.0020 & $0.00(0.00-0.26)$ & $0.00(0.00-0.87)$ & $0.00(0.00-0.31)$ & 0.1584 \\
\hline Regular physical activity & & & & 0.006 & & & & 0.1405 \\
\hline Yes & $161(36.67)$ & $174(39.64)$ & $201(45.79)$ & & $171(37.34)$ & $208(45.41)$ & $193(42.14)$ & \\
\hline No & $278(63.33)$ & $265(60.36)$ & $238(54.21)$ & & $287(62.66)$ & $250(54.59)$ & $265(57.86)$ & \\
\hline Family history of diabetes & & & & 0.0535 & & & & 0.6564 \\
\hline Yes & $3(0.68)$ & $7(1.59)$ & $10(2.28)$ & & $11(2.40)$ & $7(1.53)$ & $13(2.84)$ & \\
\hline No & $436(99.32)$ & $432(98.41)$ & $429(97.72)$ & & $447(97.60)$ & $451(98.47)$ & $445(97.16)$ & \\
\hline Body mass index $\left(\mathrm{kg} / \mathrm{m}^{2}\right)$ & $21.16 \pm 2.04$ & $23.20 \pm 1.87$ & $24.83 \pm 2.07$ & $<0.0001$ & $21.70 \pm 2.11$ & $23.53 \pm 2.04$ & $25.19 \pm 2.41$ & $<0.0001$ \\
\hline hs-CRP $(\mathrm{mg} / \mathrm{dL})^{4}$ & $0.49(0.27-1.19)$ & $0.55(0.30-1.12)$ & $0.86(0.44-1.68)$ & $<0.0001$ & $0.35(0.22-0.64)$ & $0.43(0.28-0.77)$ & $0.65(0.37-1.22)$ & $<0.0001$ \\
\hline Adiponectin $(\mu \mathrm{g} / \mathrm{mL})^{4}$ & $6.52(5.37-7.91)$ & $5.03(4.06-6.15)$ & $4.00(3.17-4.84)$ & $<0.0001$ & $8.74(7.36-10.93)$ & $6.87(5.66-8.22)$ & $5.21(4.10-6.51)$ & $<0.0001$ \\
\hline Leptin $(\mathrm{ng} / \mathrm{mL})^{4}$ & $1.01(1.01-1.11)$ & $2.15(1.64-2.70)$ & $4.15(3.21-5.76)$ & $<0.0001$ & $5.18(3.49-6.67)$ & $9.93(8.16-12.06)$ & $16.35(13.15-21.10)$ & $<0.0001$ \\
\hline LA ratio $(\mathrm{ng} / \mu \mathrm{g})^{4}$ & $0.17(0.14-0.21)$ & $0.43(0.34-0.52)$ & $1.01(0.81-1.41)$ & $<0.0001$ & $0.63(0.40-0.80)$ & $1.46(1.20-1.74)$ & $3.00(2.46-3.99)$ & $<0.0001$ \\
\hline
\end{tabular}


Table 1. Cont.

\begin{tabular}{|c|c|c|c|c|c|c|c|c|}
\hline & \multicolumn{4}{|c|}{ Men } & \multicolumn{4}{|c|}{ Women } \\
\hline & \multicolumn{3}{|c|}{ Tertile of LA Ratio $^{1}$} & \multicolumn{5}{|c|}{ Tertile of LA Ratio } \\
\hline & $\begin{array}{l}\text { T1 (Lowest) } \\
(\mathrm{n}=439)\end{array}$ & $\begin{array}{c}\text { T2 } \\
(n=439)\end{array}$ & $\begin{array}{l}\text { T3 (Highest) } \\
\text { (n = 439) }\end{array}$ & $P^{3}$ & $\begin{array}{l}\text { T1 (Lowest) } \\
(\mathrm{n}=458)\end{array}$ & $\begin{array}{c}\text { T2 } \\
(n=458)\end{array}$ & $\begin{array}{l}\text { T3 (Highest) } \\
\text { (n= 458) }\end{array}$ & $P$ \\
\hline \multicolumn{9}{|l|}{ MetS components, n (\%) } \\
\hline Abdominal obesity & $8(1.82)$ & $39(8.88)$ & $84(19.13)$ & $<0.0001$ & $31(6.77)$ & $55(12.01)$ & $94(20.52)$ & $<0.0001$ \\
\hline Elevated BP & $97(22.10)$ & $126(28.70)$ & $108(24.60)$ & 0.3923 & $108(23.58)$ & $71(15.50)$ & $77(16.81)$ & 0.0085 \\
\hline Elevated FBG & $58(13.21)$ & 77 (17.54) & $100(22.78)$ & 0.0002 & $19(4.15)$ & $20(4.37)$ & $31(6.77)$ & 0.0715 \\
\hline Elevated TG & $35(7.97)$ & 77 (17.54) & $125(28.47)$ & $<0.0001$ & $17(3.71)$ & $47(10.26)$ & $73(15.94)$ & $<0.0001$ \\
\hline Low HDL-C & $92(20.96)$ & $125(28.47)$ & $134(30.52)$ & 0.0014 & $207(45.20)$ & $252(55.02)$ & $272(59.39)$ & $<0.0001$ \\
\hline $\begin{array}{l}\text { No. of MetS components } \\
\text { at baseline }\end{array}$ & & & & $<0.0001$ & & & & $<0.0001$ \\
\hline 0 & $210(47.84)$ & $118(26.88)$ & $73(16.63)$ & & $165(36.03)$ & $127(27.73)$ & $85(18.56)$ & \\
\hline 1 & $168(38.27)$ & $198(45.10)$ & $181(41.23)$ & & $204(44.54)$ & $217(47.38)$ & $199(43.45)$ & \\
\hline 2 & $61(13.90)$ & $123(28.02)$ & $185(42.14)$ & & $89(19.43)$ & $114(24.89)$ & $174(37.99)$ & \\
\hline
\end{tabular}

LA ratio, leptin-adiponectin ratio; T, tertile; hs-CRP, high-sensitivity C-reactive protein; MetS, metabolic syndrome; BP, blood pressure; FBG, fasting blood glucose; TG, triglyceride;

HDL-C, high-density lipoprotein cholesterol. ${ }^{1}$ Cut-offs for tertiles $1-3$ of LA ratio as follows: $<0.263,0.263-0.643$, and $>0.643 \mathrm{ng} / \mu \mathrm{g}$ in men and $<0.990,0.990-2.016$, and $>2.016 \mathrm{ng} / \mu \mathrm{g}$ in women, respectively. ${ }^{2}$ Values are number (percentage) for categorical variables and mean \pm standard deviation for continuous variables. ${ }^{3} P$ values based on chi-square tests for categorical variables and generalized linear regressions for continuous variables. ${ }^{4}$ Values are presented as the median (lower quartile-upper quartile), and the $P$ values were from the Kruskal-Wallis tests. 
Table 2. Adjusted hazard ratios (with 95\% confidence intervals) for metabolic syndrome according to baseline serum adiponectin, leptin levels, and leptin-adiponectin ratio in Korean men aged 45-76 years.

\begin{tabular}{|c|c|c|c|c|c|c|}
\hline & \multicolumn{3}{|c|}{ Tertile } & \multicolumn{3}{|c|}{ Per 1 SD } \\
\hline & T1 (Lowest) & T2 & T3 (Highest) & $P_{\text {trend }}{ }^{2}$ & Increment & $P$ \\
\hline & HR $(95 \%$ CI) & HR (95\% CI) & HR $(95 \%$ CI) & & HR $(95 \%$ CI) & \\
\hline \multicolumn{7}{|c|}{ Adiponectin } \\
\hline Person-years & 2748 & 2947 & 3075 & & 8770 & \\
\hline Incident cases $(n)$ & 167 & 113 & 79 & & 359 & \\
\hline Rate per & 60.8 & 38.3 & 25.7 & & 40.9 & \\
\hline Median & 3.5 & 5.1 & 7.3 & & 5.1 & \\
\hline Ranges & $0.48-4.34$ & $4.36-5.93$ & $5.94-25.04$ & & $0.48-25.04$ & \\
\hline Model 11 & 1.00 & $0.62(0.49-0.79)$ & $0.39(0.30-0.52)$ & $<0.0001$ & $0.85(0.80-0.90)$ & $<0.0001$ \\
\hline Model 2 & 1.00 & $0.71(0.56-0.91)$ & $0.53(0.40-0.70)$ & $<0.0001$ & $0.90(0.85-0.95)$ & 0.0003 \\
\hline \multicolumn{7}{|c|}{ Leptin } \\
\hline Person-years & 3145 & 2951 & 2674 & & 8770 & \\
\hline Incident cases $(n)$ & 55 & 119 & 185 & & 359 & \\
\hline $\begin{array}{l}\text { Rate per } 1000 \\
\text { person-years }\end{array}$ & 17.5 & 40.3 & 69.2 & & 40.9 & \\
\hline Median & 1.0 & 2.1 & 4.3 & & 2.1 & \\
\hline Ranges & $1.01-1.37$ & $1.38-2.91$ & $2.91-24.08$ & & $1.01-24.08$ & \\
\hline Model 1 & 1.00 & $2.39(1.73-3.29)$ & $4.11(3.04-5.57)$ & $<0.0001$ & $1.14(1.11-1.17)$ & $<0.0001$ \\
\hline Model 2 & 1.00 & $1.95(1.39-2.74)$ & $2.88(2.01-4.13)$ & $<0.0001$ & $1.05(1.01-1.08)$ & 0.0176 \\
\hline \multicolumn{7}{|c|}{ LA ratio } \\
\hline Person-years & 3150 & 2953 & 2667 & & 8770 & \\
\hline Incident cases $(n)$ & 51 & 124 & 184 & & 359 & \\
\hline $\begin{array}{l}\text { Rate per } 1000 \\
\text { person-years }\end{array}$ & 16.2 & 42.0 & 69.0 & & 40.9 & \\
\hline Median & 0.2 & 0.4 & 1.0 & & 0.4 & \\
\hline Ranges & $0.1-0.3$ & $0.3-0.6$ & $0.6-5.3$ & & $0.1-5.3$ & \\
\hline Model 1 & 1.00 & $2.72(1.96-3.77)$ & 4.66 (3.39-6.39) & $<0.0001$ & $1.74(1.56-1.94)$ & $<0.0001$ \\
\hline Model 2 & 1.00 & $2.16(1.53-3.03)$ & $3.07(2.13-4.44)$ & $<0.0001$ & $1.40(1.22-1.62)$ & $<0.0001$ \\
\hline
\end{tabular}

T, tertile; HR, hazard ratio; CI, confidence interval; LA ratio, leptin-adiponectin ratio; BMI, body mass index; hs-CRP, high-sensitivity C-reactive protein. ${ }^{1}$ Model 1 was adjusted for age (years); model 2 was additionally adjusted for area of residence (Ansan or Ansung), education level ( $\leq$ elementary school, middle/high school, or $\geq$ college), smoking status (never, past, or current), alcohol consumption (g/day), regular physical activity (yes or no), BMI $\left(\mathrm{kg} / \mathrm{m}^{2}\right)$, family history of diabetes (yes or no), and serum hs-CRP level $(\mathrm{mg} / \mathrm{L}) .{ }^{2}$ Tests for trend linearity were conducted with the Wald test by considering the median values of each tertile of adiponectin, leptin, and LA ratio as continuous variables in the analytical models. 
Table 3. Adjusted hazard ratios (with $95 \%$ confidence intervals) for metabolic syndrome according to baseline serum adiponectin, leptin levels, and leptin-adiponectin ratio in Korean women aged 45-76 years.

\begin{tabular}{|c|c|c|c|c|c|c|}
\hline & \multicolumn{3}{|c|}{ Tertile } & \multirow{3}{*}{$P_{\text {trend }}{ }^{2}$} & Per 1 SD & \multirow{3}{*}{$P$} \\
\hline & T1 (Lowest) & $\mathrm{T} 2$ & T3 (Highest) & & Increment & \\
\hline & HR $(95 \%$ CI) & HR (95\% CI) & HR (95\% CI) & & HR $(95 \%$ CI) & \\
\hline \multicolumn{7}{|c|}{ Adiponectin } \\
\hline Person-years & 3069 & 3132 & 3191 & & 9392 & \\
\hline Incident cases $(n)$ & 154 & 131 & 100 & & 385 & \\
\hline $\begin{array}{l}\text { Rate per } 1000 \\
\text { person-years }\end{array}$ & 50.2 & 41.8 & 31.3 & & 41.0 & \\
\hline Median & 4.7 & 6.9 & 9.6 & & 6.9 & \\
\hline Ranges & $0.87-5.88$ & $5.88-8.09$ & $8.10-27.78$ & & $0.9-27.8$ & \\
\hline Model $1^{1}$ & 1.00 & $0.81(0.64-1.02)$ & $0.51(0.39-0.66)$ & $<0.0001$ & $0.90(0.86-0.93)$ & $<0.0001$ \\
\hline Model 2 & 1.00 & $0.82(0.65-1.03)$ & $0.54(0.42-0.71)$ & $<0.0001$ & $0.91(0.88-0.95)$ & $<0.0001$ \\
\hline \multicolumn{7}{|c|}{ Leptin } \\
\hline Person-years & 3296 & 3145 & 2951 & & 9392 & \\
\hline Incident cases $(n)$ & 86 & 128 & 171 & & 385 & \\
\hline $\begin{array}{l}\text { Rate per } 1000 \\
\text { person-years }\end{array}$ & 26.1 & 40.7 & 57.9 & & 41.0 & \\
\hline Median & 5.1 & 9.7 & 17.1 & & 9.7 & \\
\hline Ranges & $1.01-7.33$ & $7.37-12.62$ & $12.65-52.36$ & & $1.0-52.4$ & \\
\hline Model 1 & 1.00 & $1.79(1.36-2.36)$ & $2.47(1.90-3.21)$ & $<0.0001$ & $1.04(1.03-1.05)$ & $<0.0001$ \\
\hline Model 2 & 1.00 & $1.45(1.09-1.94)$ & $1.55(1.13-2.13)$ & $<0.0001$ & $1.01(0.99-1.02)$ & 0.4084 \\
\hline \multicolumn{7}{|c|}{ LA ratio } \\
\hline Person-years & 3273 & 3169 & 2950 & & 9392 & \\
\hline Incident cases $(n)$ & 88 & 120 & 177 & & 385 & \\
\hline $\begin{array}{l}\text { Rate per } 1000 \\
\text { person-years }\end{array}$ & 26.9 & 37.9 & 60.0 & & 41.0 & \\
\hline Median & 5.1 & 9.7 & 17.1 & & 1.5 & \\
\hline Ranges & $1.0-7.3$ & $7.4-12.6$ & $12.6-52.4$ & & $0.1-24.6$ & \\
\hline Model 1 & 1.00 & $1.67(1.26-2.20)$ & $2.78(2.14-3.62)$ & $<0.0001$ & $1.11(1.08-1.15)$ & $<0.0001$ \\
\hline Model 2 & 1.00 & $1.37(1.02-1.84)$ & $1.94(1.41-2.66)$ & $<0.0001$ & $1.04(0.99-1.09)$ & 0.1096 \\
\hline
\end{tabular}

T, tertile; HR, hazard ratio; CI, confidence interval; LA ratio, leptin-adiponectin ratio; BMI, body mass index; hs-CRP, high-sensitivity C-reactive protein. ${ }^{1}$ Model 1 was adjusted for age (years); model 2 was additionally adjusted for area of residence (Ansan or Ansung), education level (selementary school, middle/high school, or $\geq$ college), smoking status (never, past, or current), alcohol consumption (g/day), regular physical activity (yes or no), BMI $\left(\mathrm{kg} / \mathrm{m}^{2}\right)$, family history of diabetes (yes or no), and serum hs-CRP level $(\mathrm{mg} / \mathrm{L}) .{ }^{2}$ Tests for trend linearity were conducted with the Wald test by considering the median values of each tertile of adiponectin, leptin, and LA ratio as continuous variables in the analytical models.

Supplementary Tables S3 and S4 show the sex-specific associations between levels of adiponectin, leptin, and the LA ratio with the incident MetS components. Higher baseline adiponectin level showed a 35\% and 58\% reduction in the risk of elevated TG in men and women, respectively, but an inverse association between low HDL-C and MetS incidence was found only in men. A higher leptin level at baseline was associated with an increased risk of abdominal obesity and elevated BP in both men and women. In the sex-stratified analysis, leptin level was associated with a higher incidence of elevated FBG, elevated TG, and low HDL-C in men only. Differences between the sexes regarding LA ratio and the risk of developing individual MetS components were also observed. Among men, increasing LA ratio was associated with an elevated risk of abdominal obesity, elevated FBG, and low HDL-C, while among women, LA ratio was associated with an increased risk of abdominal obesity, elevated BP, and elevated TG.

Figure 2 shows MetS incidence in each group of cross-classified tertiles by adiponectin and leptin levels. Compared to the combination of high adiponectin-low leptin levels, the combination of low adiponectin-high leptin levels showed the greatest estimated risk among both men (HR: 5.74, 95\% CI: 3.30-9.98) and women (HR: 2.77, 95\% CI: 1.75-4.38). The test for interaction between tertiles of adiponectin and leptin was not statistically significant $\left(P_{\text {interaction }}>0.05\right)$. 

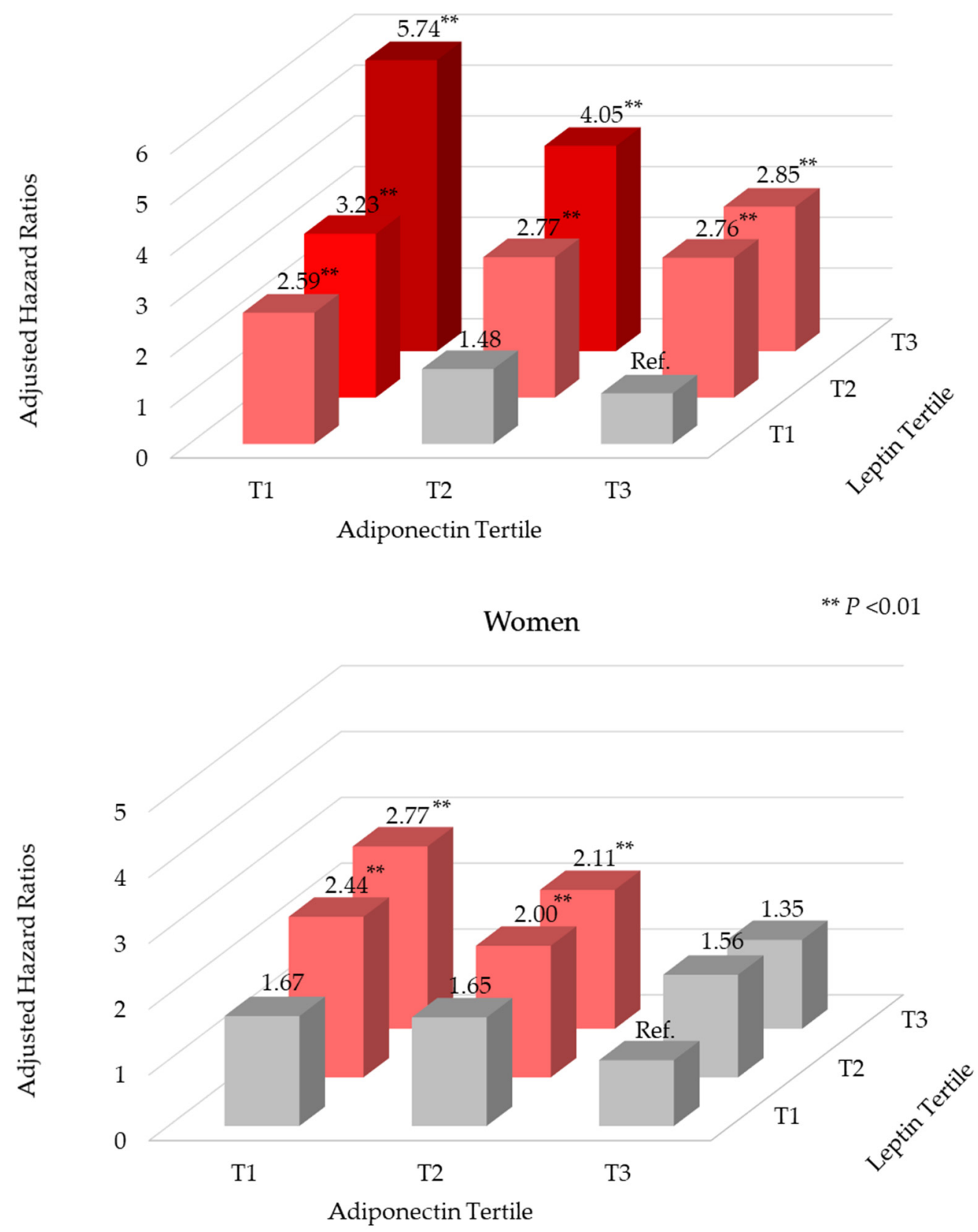

Figure 2. Association of metabolic syndrome by baseline adiponectin and leptin level strata in Korean adults aged $45-76$ years ${ }^{1} .{ }^{1}$ Model was adjusted for age (years), area of residence (Ansan or Ansung), education level ( $\leq$ elementary school, middle/high school, or $\geq$ college), smoking status (never, past, or current), alcohol consumption (g/day), regular physical activity (yes or no), BMI ( $\mathrm{kg} / \mathrm{m}^{2}$ ), family history of diabetes (yes or no), and serum hs-CRP level (mg/L).

\section{Discussion}

Middle-aged and older Koreans in the KoGES Ansan-Ansung cohort study were observed for significant relationships between serum adiponectin and leptin levels and LA ratio, and the future risk of developing MetS or its individual components. In the present study, an association between higher adiponectin levels and the lowered incidence of MetS was shown. In contrast, elevated leptin levels and a higher LA ratio were positively associated with risk of MetS development. When examining 
the combined effect of serum adiponectin and leptin levels by using cross-classified tertiles, the low adiponectin-high leptin combination foretold the greatest risk of developing MetS.

Adiponectin plays a critical role in insulin sensitivity and inflammation, and thus hypoadiponectinemia is thought to be linked with multiple metabolic risk factors [27]. Previous findings from epidemiological studies compare well with our results, indicating an inverse association between adiponectin levels and MetS risk. A cross-sectional analysis of a Chinese population found that those in the highest adiponectin quartile showed an increased MetS risk (odds ratio (OR): 3.38, 95\% CI: 2.56-4.46) over those in the lowest quartile [28]. Another cross-sectional study of middle-aged Korean adults compared the first adiponectin quartile with those of the fourth quartile and found a decreased risk of MetS (OR: 0.32, 95\% CI: 0.20-0.50 for men; OR: 0.57, 95\% CI: $0.43-0.76$ for women) [19]. Contrary to adiponectin, elevated leptin levels and hyperleptinemia have been demonstrated to be closely associated with metabolic abnormalities [29,30]. For example, a prospective cohort study of 748 middle-aged white adults reported that baseline leptin levels accurately predicted the future risk of developing MetS and obesity [31]. In overweight and obese European adolescents, increased leptin level was associated with an increased prevalence of MetS [32]. Adiponectin and leptin are produced primarily by adipose tissue [8,33] and are related to measures of obesity, such as BMI [34]. It has been seen that adiponectin levels are lowered and leptin levels are elevated in those with obesity $[35,36]$. Previous studies revealed that serum adiponectin and leptin levels were significant risk factors for MetS in a Western population with high adiposity. We extend these findings to a Korean population, with a relatively lower adiposity compared to the Western population.

We found that LA ratio was a more accurate marker than adiponectin or leptin alone for identification of individuals at risk for MetS in the Korean adult population. In parallel with our findings, previous investigations have reported the utility of LA ratio in the early diagnosis of MetS. A study involving 2046 Chinese men and women aged 60-96 years indicated that one SD increase of LA ratio was associated with an 83\% (OR: 1.83, 95\% CI: 1.38-2.45, $P<0.001$ ) and 132\% (OR: 2.32, 95\% CI: $1.82-2.96, P<0.001$ ) higher risk of MetS in men and women, respectively [17]. In addition, receiver operating characteristic curve analysis confirmed that the use of LA ratio significantly improved diagnostic ability by $9.6 \%$ in men and $7.7 \%$ in women compared to adiponectin alone. Another study based on a French population found that LA ratio was positively correlated with the number of MetS cases, and with each component thereof [37]. Mirza et al. also reported that adiponectin-leptin ratio had a greater diagnostic ability for MetS compared with either adiponectin or leptin alone [38]. Our findings suggest that adiponectin, leptin, and LA ratio might be promising biomarkers for predicting the future risk of MetS in the middle-aged and older Korean adults, particularly in those with one or more MetS risk factors. The importance of early detection of diseases has been recognized in prevention and timely management. Investigations have been made to identify useful biomarkers that enable early diagnosis of diseases [39-41]. Adiponectin and leptin levels are known to be significantly associated with obesity. In the current study, we found that adiponectin, leptin, and LA ratio were prospectively associated with MetS risk among Korean adults even after adjusting for obesity, which is one of the most significant risk factors for MetS. Therefore, monitoring adiponectin and leptin in combination with the measurement of obesity may provide a simple, easy, and cost-effective approach for early detection of MetS.

In this study, adiponectin, leptin, and LA ratio were differentially associated with the risk of MetS development by sex. Although the reason for the sex difference in these associations has not been clearly elucidated, sexual dimorphism could provide a partial explanation. First, sex differences in adiposity and fat distribution via sex hormones such as androgen and estrogen could be one reason. In general, women have twice as much body fat as men, and different body fat distributions are observed between men and women. Body fat in women is mainly distributed in the gluteal-femoral area such as the hips and thighs, whereas men accumulate body fat in the abdominal or visceral area [42]. Adiponectin and leptin gene expression is more likely to occur in subcutaneous than in visceral adipose tissue [43,44]. Furthermore, serum leptin levels are affected only by leptin produced by subcutaneous adipose 
tissue [45]. This may cause the differences in levels of adiponectin and leptin seen in the blood of men and women. Second, another possible explanation is a sex difference of genetic variants of adipokine genes. Christen et al. [46] reported that a single nucleotide polymorphism near the LEP gene (rs791595) altered serum leptin levels. Carrying one LEP risk allele was associated with a $3 \mu \mathrm{g} / \mathrm{L}$ increase in leptin in women, but such an association was not observed in men. Lastly, metabolic flexibility could be a key explanation in sex differences observed in the relationships between adiponectin, leptin, and LA ratio on incident MetS. Metabolic flexibility is the ability of the human body to adapt to metabolic changes. Additionally, metabolic flexibility may be determined by how closely the transition between fat oxidation and glucose oxidation occurs to meet metabolic requirements $[47,48]$. Female skeletal muscle is optimized for oxidation and lipid storage, thus women have a greater capacity for glucose and fatty acid utilization [49]. Furthermore, it has been documented that serum adiponectin levels correlate with metabolic flexibility [50]. Adiponectin, which exists at a higher level in women than in men, may promote metabolic flexibility by increasing lipid synthesis and storage under fed conditions and enhancing lipid catabolism under fasting conditions [51]. Together, these suggest that women are more metabolically flexible than men in a metabolically challenged state.

To the best of our knowledge, this is the first prospective study investigating the associations of serum adiponectin, leptin, and LA ratio with MetS incidence. The present study has several strengths, including the population-based prospective design without exposure to recall bias, the large sample size, length of follow-up period, and controls for possible covariates that could affect the relationships between adiponectin and leptin levels, and MetS (e.g., sex, BMI, and hs-CRP concentration). However, there were also limitations that need to be mentioned. First, we estimated the associations between the three markers and MetS incidence based on a single measurement of adiponectin and leptin levels due to lack of available data. The results of this study do not provide a comprehensive explanation for the effect of alterations in adiponectin and leptin levels over time on future MetS risk. Second, we used only total adiponectin concentration. It has been well documented that high-molecular-weight adiponectin is the most active fraction of adiponectin [52] and contributes widely to insulin-sensitizing activity [53]. However, since previous epidemiological studies reported that total adiponectin and high-molecular-weight adiponectin showed a similar predictive ability in insulin resistance and metabolic abnormalities [54,55], the measurement of total adiponectin used in this study was considered sufficient. Third, baseline dietary information was not included in the statistical models due to unavailability. Nevertheless, we included alcohol consumption, which is one of the major dietary factors contributing to the serum levels of adiponectin and leptin [56-58]. Further investigation exploring interactions between diet and adiponectin and leptin levels over time on MetS risk is needed. Fourth, although we adjusted for various potential confounders related to exposure and outcome variables of interest in this study, the issue of unmeasured or unidentified confounders still remains. Moreover, our study participants consist of middle-aged and older Korean adults residing in specific areas (two cities in Gyeonggi province) of Korea. Our findings may be difficult to generalize to other Korean populations with different ages or lifestyle characteristics. Thus, additional investigations are needed to confirm the applicability of our results to the general Korean population.

\section{Conclusions}

In conclusion, higher adiponectin levels were associated with a lower risk of developing MetS, while higher leptin levels and LA ratio were associated with an elevated MetS incidence in both men and women. Our results support the role of adiponectin and leptin levels and LA ratio as useful predictive markers to measure the risk of future MetS development among middle-aged and older Koreans. Continuous monitoring of adiponectin, leptin, and LA ratio could help detect high-risk individuals for MetS. Furthermore, the present results warrant further investigation into how distinct trajectory patterns of serum adiponectin and leptin levels are associated with the risk of incident MetS in a large population-based prospective study with a longer follow-up period. 
Supplementary Materials: The following are available online at http://www.mdpi.com/1660-4601/17/9/3287/s1, Table S1: Characteristics of the study participants according to adiponectin levels in Korean men and women aged 45-76 years; Table S2: Characteristics of the study participants according to leptin levels in Korean men and women aged 45-76 years; Table S3: Adjusted hazard ratios (with 95\% confidence intervals) for each component of metabolic syndrome according to baseline serum adiponectin, leptin levels, and leptin-adiponectin ratio in Korean men aged 45-76 years; Table S4: Adjusted hazard ratios (with 95\% confidence intervals) for each component of metabolic syndrome according to baseline serum adiponectin, leptin levels, and leptin-adiponectin ratio in Korean women aged $45-76$ years.

Author Contributions: Conceptualization, K.W.L. and D.S.; methodology, K.W.L.; statistical analysis, K.W.L.; data interpretation, K.W.L. and D.S.; writing-original draft preparation, K.W.L. and D.S.; writing-review and editing, K.W.L. and D.S.; funding acquisition, K.W.L. All authors have read and agreed to the published version of the manuscript.

Funding: This study was conducted with research funds from Gwangju University in 2020.

Acknowledgments: Epidemiological data in this study were obtained from the Korean Genome and Epidemiology Study by the National Institute of Health, Centers for Disease Control and Prevention, Ministry for Health and Welfare, Republic of Korea.

Conflicts of Interest: The authors declare no conflict of interest.

\section{References}

1. Naghavi, M.; Abajobir, A.A.; Abbafati, C.; Abbas, K.M.; Abd-Allah, F.; Abera, S.F.; Aboyans, V.; Adetokunboh, O.; Afshin, A.; Agrawal, A.; et al. Global, regional, and national age-sex specific mortality for 264 causes of death, 1980-2016: A systematic analysis for the Global Burden of Disease Study 2016. Lancet 2017, 390, 1151-1210. [CrossRef]

2. Korea Centers for Disease Control and Prevention. Fact book: Non-communicable Diseases 2019. Cheongju: Korea Centers for Disease Control and Prevention. 2019. Available online: http://nih.go.kr/gallery.es?mid=a20503020000\&bid=0003\&b_list=9\&act=view\&list_no=144581\& nPage $=1 \&$ list_no_npage $=2 \&$ keyField $=\&$ keyWord $=\&$ orderby $=($ accessed on 10 January 2020).

3. Grundy, S.M.; Brewer, H.B., Jr.; Cleeman, J.I.; Smith, S.C., Jr.; Lenfant, C. Definition of metabolic syndrome: Report of the national heart, lung, and blood institute/American heart association conference on scientific issues related to definition. Circulation 2004, 109, 433-438. [CrossRef]

4. Korean Society of Cardiometabolic Syndrome. Metabolic syndrome fact sheet in Korea. 2018. Available online: http://kscms.org/en/uploads/Metabolic_Syndrome.pdf (accessed on 8 January 2020).

5. Grundy, S.M. Metabolic syndrome: A multiplex cardiovascular risk factor. J. Clin. Endocrinol. Metab. 2007, 92, 399-404. [CrossRef]

6. Haffner, S.M. The metabolic syndrome: Inflammation, diabetes mellitus, and cardiovascular disease. Am. J. Cardiol. 2006, 97, 3-11. [CrossRef]

7. Gómez-Ambrosi, J.; Catalán, V.; Rodríguez, A.; Andrada, P.; Ramírez, B.; Ibáñez, P.; Vila, N.; Romero, S.; Margall, M.A.; Gil, M.J.; et al. Increased cardiometabolic risk factors and inflammation in adipose tissue in obese subjects classified as metabolically healthy. Diabetes Care 2014, 37, 2813-2821. [CrossRef]

8. Scherer, P.E.; Williams, S.; Fogliano, M.; Baldini, G.; Lodish, H.F. A novel serum protein similar to C1q, produced exclusively in adipocytes. J. Biol. Chem. 1995, 270, 26746-26749. [CrossRef]

9. Kadowaki, T.; Yamauchi, T.; Kubota, N.; Hara, K.; Ueki, K.; Tobe, K. Adiponectin and adiponectin receptors in insulin resistance, diabetes, and the metabolic syndrome. J. Clin. Investig. 2006, 116, 1784-1792. [CrossRef]

10. Weyer, C.; Funahashi, T.; Tanaka, S.; Hotta, K.; Matsuzawa, Y.; Pratley, R.E.; Tataranni, P.A. Hypoadiponectinemia in obesity and type 2 diabetes: Close association with insulin resistance and hyperinsulinemia. J. Clin. Endocrinol. Metab. 2001, 86, 1930-1935. [CrossRef]

11. Im, J.-A.; Kim, S.-H.; Lee, J.-W.; Shim, J.-Y.; Lee, H.-R.; Lee, D.-C. Association between hypoadiponectinemia and cardiovascular risk factors in nonobese healthy adults. Metabolism 2006, 55, 1546-1550. [CrossRef]

12. Friedman, J. Leptin at 20: An overview. J. Endocrinol. 2014, 223, T1-T8. [CrossRef]

13. Da Paz Filho, G.; Mastronardi, C.; Franco, C.; Wang, K.; Wong, M.-L.; Licinio, J. Leptin: Molecular mechanisms, systemic pro-inflammatory effects, and clinical implications [Leptina: Mecanismos moleculares, efeitos proinflamatorios sistmicos e implicoes clinicas]. Arquivos Bras. Endocrinol. Metabol. 2012, 56, 597-607. [CrossRef] 
14. Kumada, M.; Kihara, S.; Sumitsuji, S.; Kawamoto, T.; Matsumoto, S.; Ouchi, N.; Arita, Y.; Okamoto, Y.; Shimomura, I.; Hiraoka, H.; et al. Association of hypoadiponectinemia with coronary artery disease in men. Arterioscler. Thromb. Vasc. Biol. 2003, 23, 85-89. [CrossRef]

15. de Courten, M.; Zimmet, P.; Hodge, A.; Collins, V.; Nicolson, M.; Staten, M.; Dowse, G.; Alberti, K. Hyperleptinaemia: The missing link in the metabolic syndrome? Diabet. Med. 1997, 14, 200-208. [CrossRef]

16. Lindberg, S.; Jensen, J.S.; Bjerre, M.; Frystyk, J.; Flyvbjerg, A.; Jeppesen, J.; Mogelvang, R. Low adiponectin levels at baseline and decreasing adiponectin levels over 10 years of follow-up predict risk of the metabolic syndrome. Diabetes Metab. 2017, 43, 134-139. [CrossRef]

17. Zhuo, Q.; Wang, Z.; Fu, P.; Piao, J.; Tian, Y.; Xu, J.; Yang, X. Comparison of adiponectin, leptin and leptin to adiponectin ratio as diagnostic marker for metabolic syndrome in older adults of Chinese major cities. Diabetes Res. Clin. Pract. 2009, 84, 27-33. [CrossRef]

18. Zamboni, M.; Zoico, E.; Fantin, F.; Panourgia, M.P.; Di Francesco, V.; Tosoni, P.; Solerte, B.; Vettor, R.; Bosello, O. Relation between leptin and the metabolic syndrome in elderly women. J. Gerontol. Ser. A Biol. Sci. Med. Sci. 2004, 59, M396-M400. [CrossRef]

19. Koh, S.-B.; Yoon, J.; Kim, J.-Y.; Yoo, B.-S.; Lee, S.-H.; Park, J.-K.; Choe, K.-H. Relationships between serum adiponectin with metabolic syndrome and components of metabolic syndrome in non-diabetic Koreans: ARIRANG study. Yonsei Med. J. 2011, 52, 234-241. [CrossRef]

20. Yun, J.E.; Kimm, H.; Jo, J.; Jee, S.H. Serum leptin is associated with metabolic syndrome in obese and nonobese Korean populations. Metabolism 2010, 59, 424-429. [CrossRef]

21. Choi, K.M.; Lee, J.; Lee, K.; Seo, J.A.; Oh, J.; Kim, S.G.; Kim, N.H.; Choi, D.; Baik, S.-H. Serum adiponectin concentrations predict the developments of type 2 diabetes and the metabolic syndrome in elderly Koreans. Clin. Endocrinol. 2004, 61, 75-80. [CrossRef]

22. Kang, D.R.; Yadav, D.; Koh, S.-B.; Kim, J.-Y.; Ahn, S.V. Impact of serum leptin to adiponectin ratio on regression of metabolic syndrome in high-risk individuals: The ARIRANG study. Yonsei Med. J. 2017, 58, 339-346. [CrossRef]

23. Correia, M.; Haynes, W. Lessons from leptin's molecular biology: Potential therapeutic actions of recombinant leptin and leptin-related compounds. Mini Rev. Med. Chem. 2007, 7, 31-38. [CrossRef]

24. Kim, Y.; Han, B.-G.; Group, K. Cohort profile: The Korean genome and epidemiology study (KoGES) consortium. Int. J. Epidemiol. 2017, 46, e20. [CrossRef]

25. Expert Panel on Detection, E. Executive summary of the third report of the National Cholesterol Education Program (NCEP) expert panel on detection, evaluation, and treatment of high blood cholesterol in adults (Adult Treatment Panel III). JAMA 2001, 285, 2486.

26. Alberti, K.; Eckel, R.H.; Grundy, S.M.; Zimmet, P.Z.; Cleeman, J.I.; Donato, K.A.; Fruchart, J.-C.; James, W.P.T.; Loria, C.M.; Smith, S.C., Jr.; et al. Harmonizing the metabolic syndrome: A joint interim statement of the international diabetes federation task force on epidemiology and prevention; national heart, lung, and blood institute; American heart association; world heart federation; international atherosclerosis society; and international association for the study of obesity. Circulation 2009, 120, 1640-1645.

27. Matsuzawa, Y.; Funahashi, T.; Kihara, S.; Shimomura, I. Adiponectin and metabolic syndrome. Arterioscler. Thromb. Vasc. Biol. 2004, 24, 29-33. [CrossRef]

28. Wang, J.; Li, H.; Franco, O.H.; Yu, Z.; Liu, Y.; Lin, X. Adiponectin and metabolic syndrome in middle-aged and elderly Chinese. Obesity 2008, 16, 172-178. [CrossRef]

29. Ghadge, A.A.; Khaire, A.A. Leptin as a predictive marker for metabolic syndrome. Cytokine 2019, 121, 154735. [CrossRef]

30. Chen, M.-C.; Hsu, B.-G.; Lee, C.-J.; Wang, J.-H. Hyperleptinemia positively correlates with cardiometabolic syndrome in hypertensive patients. Int. J. Clin. Exp. Pathol. 2016, 9, 12959-12967.

31. Franks, P.W.; Brage, S.; Luan, J.A.; Ekelund, U.; Rahman, M.; Farooqi, I.S.; Halsall, I.; O’Rahilly, S.; Wareham, N.J. Leptin predicts a worsening of the features of the metabolic syndrome independently of obesity. Obes. Res. 2005, 13, 1476-1484. [CrossRef]

32. Nappo, A.; Gonzalez-Gil, E.; Ahrens, W.; Bammann, K.; Michels, N.; Moreno, L.; Kourides, Y.; Iacoviello, L.; Mårild, S.; Fraterman, A.; et al. Analysis of the association of leptin and adiponectin concentrations with metabolic syndrome in children: Results from the IDEFICS study. Nutr. Metab. Cardiovasc. Dis. 2017, 27, 543-551. [CrossRef] 
33. Zhang, Y.; Proenca, R.; Maffei, M.; Barone, M.; Leopold, L.; Friedman, J.M. Positional cloning of the mouse obese gene and its human homologue. Nature 1994, 372, 425-432. [CrossRef] [PubMed]

34. Ruhl, C.E.; Everhart, J.E. Leptin concentrations in the United States: Relations with demographic and anthropometric measures. Am. J. Clin. Nutr. 2001, 74, 295-301. [CrossRef] [PubMed]

35. Matsubara, M.; Maruoka, S.; Katayose, S. Inverse relationship between plasma adiponectin and leptin concentrations in normal-weight and obese women. Eur. J. Endocrinol. 2002, 147, 173-180. [CrossRef]

36. Silha, J.V.; Krsek, M.; Skrha, J.V.; Sucharda, P.; Nyomba, B.; Murphy, L.J. Plasma resistin, adiponectin and leptin levels in lean and obese subjects: Correlations with insulin resistance. Eur. J. Endocrinol. 2003, 149, 331-336. [CrossRef]

37. Gauthier, A.; Dubois, S.; Bertrais, S.; Gallois, Y.; Aube, C.; Gagnadoux, F. The leptin to adiponectin ratio is a marker of the number of metabolic syndrome criteria in French adults. J. Metab. Syndr. 2012, 1, 101. [CrossRef]

38. Mirza, S.; Qu, H.-Q.; Li, Q.; Martinez, P.J.; Rentfro, A.R.; McCormick, J.B.; Fisher-Hoch, S.P. Adiponectin/leptin ratio and metabolic syndrome in a Mexican American population. Clin. Investig. Med. Med. Clin. Exp. 2011, 34, E290. [CrossRef]

39. Falahi, E.; Rad, A.H.; Roosta, S. What is the best biomarker for metabolic syndrome diagnosis? Diabetes Metab. Syndr. 2015, 9, 366-372. [CrossRef]

40. Mayeux, R. Biomarkers: Potential uses and limitations. NeuroRx 2004, 1, 182-188. [CrossRef]

41. O'Neill, S.; Bohl, M.; Gregersen, S.; Hermansen, K.; O’Driscoll, L. Blood-based biomarkers for metabolic syndrome. Trends Endrocrinol. Metab. 2016, 27, 363-374. [CrossRef]

42. Karastergiou, K.; Smith, S.R.; Greenberg, A.S.; Fried, S.K. Sex differences in human adipose tissues-The biology of pear shape. Biol. Sex Differ. 2012, 3, 13. [CrossRef]

43. Lihn, A.S.; Bruun, J.M.; He, G.; Pedersen, S.B.; Jensen, P.F.; Richelsen, B. Lower expression of adiponectin mRNA in visceral adipose tissue in lean and obese subjects. Mol. Cell. Endocrinol. 2004, 219, 9-15. [CrossRef]

44. Masuzaki, H.; Ogawa, Y.; Isse, N.; Satoh, N.; Okazaki, T.; Shigemoto, M.; Mori, K.; Tamura, N.; Hosoda, K.; Yoshimasa, Y.; et al. Human obese gene expression: Adipocyte-specific expression and regional differences in the adipose tissue. Diabetes 1995, 44, 855-858. [CrossRef]

45. Van Harmelen, V.; Reynisdottir, S.; Eriksson, P.; Thörne, A.; Hoffstedt, J.; Lönnqvist, F.; Arner, P. Leptin secretion from subcutaneous and visceral adipose tissue in women. Diabetes 1998, 47, 913-917. [CrossRef]

46. Christen, T.; Trompet, S.; Noordam, R.; van Klinken, J.B.; van Dijk, K.W.; Lamb, H.J.; Cobbaert, C.M.; den Heijer, M.; Jazet, I.M.; Jukema, J.W.; et al. Sex differences in body fat distribution are related to sex differences in serum leptin and adiponectin. Peptides 2018, 107, 25-31. [CrossRef]

47. Storlien, L.; Oakes, N.D.; Kelley, D.E. Metabolic flexibility. Proc. Nutr. Soc. 2004, 63, 363-368. [CrossRef]

48. Bouchard, C.; Despres, J.-P.; Mauriège, P. Genetic and nongenetic determinants of regional fat distribution. Endocr. Rev. 1993, 14, 72-93. [CrossRef]

49. Sparks, L.M.; Pasarica, M.; Sereda, O.; dejonge, L.; Thomas, S.; Loggins, H.; Xie, H.; Miles, J.M.; Smith, S.R. Effect of adipose tissue on the sexual dimorphism in metabolic flexibility. Metabolism 2009, 58, 1564-1571. [CrossRef]

50. Ong, K.K.; Frystyk, J.; Flyvbjerg, A.; Petry, C.J.; Ness, A.; Dunger, D.B. Sex-discordant associations with adiponectin levels and lipid profiles in children. Diabetes 2006, 55, 1337-1341. [CrossRef]

51. Asterholm, I.W.; Scherer, P.E. Enhanced metabolic flexibility associated with elevated adiponectin levels. Am. J. Pathol. 2010, 176, 1364-1376. [CrossRef]

52. Berg, A.H.; Combs, T.P.; Scherer, P.E. ACRP30/adiponectin: An adipokine regulating glucose and lipid metabolism. Trends Endocrinol. Metab. 2002, 13, 84-89. [CrossRef]

53. Rasouli, N.; Kern, P.A. Adipocytokines and the metabolic complications of obesity. J. Clin. Endocrinol. Metab. 2008, 93, s64-s73. [CrossRef] [PubMed]

54. Blüher, M.; Brennan, A.M.; Kelesidis, T.; Kratzsch, J.; Fasshauer, M.; Kralisch, S.; Williams, C.J.; Mantzoros, C.S. Total and high-molecular weight adiponectin in relation to metabolic variables at baseline and in response to an exercise treatment program: Comparative evaluation of three assays. Diabetes Care 2007, 30, 280-285. [CrossRef] [PubMed]

55. Nakashima, R.; Yamane, K.; Kamei, N.; Nakanishi, S.; Kohno, N. Low serum levels of total and high-molecular-weight adiponectin predict the development of metabolic syndrome in Japanese-Americans. J. Endocrinol. Investig. 2011, 34, 615-619. 
56. Sierksma, A.; Patel, H.; Ouchi, N.; Kihara, S.; Funahashi, T.; Heine, R.J.; Grobbee, D.E.; Kluft, C.; Hendriks, H.F. Effect of moderate alcohol consumption on adiponectin, tumor necrosis factor- $\alpha$, and insulin sensitivity. Diabetes Care 2004, 27, 184-189. [CrossRef] [PubMed]

57. Pravdova, E.; Macho, L.; Fickova, M. Alcohol intake modifies leptin, adiponectin and resistin serum levels and their mRNA expressions in adipose tissue of rats. Endocr. Regul. 2009, 43, 117-125. [PubMed]

58. Pischon, T.; Girman, C.J.; Rifai, N.; Hotamisligil, G.S.; Rimm, E.B. Association between dietary factors and plasma adiponectin concentrations in men. Am. J. Clin. Nutr. 2005, 81, 780-786. [CrossRef]

(C) 2020 by the authors. Licensee MDPI, Basel, Switzerland. This article is an open access article distributed under the terms and conditions of the Creative Commons Attribution (CC BY) license (http://creativecommons.org/licenses/by/4.0/). 